\title{
Uso de técnicas de regressão na avaliação, em bovinos de corte, da eficiência de conversão do alimento em produto: proposição de método e significância nutricional ${ }^{1}$
}

\section{Edenio Detmann², Mateus Pies Gionbelli³, Sebastião de Campos Valadares Filho², Pedro Veiga Rodrigues Paulino ${ }^{2}$}

\footnotetext{
${ }^{1}$ Trabalho conduzido com apoio do CNPq, FAPEMIG (PPM) e INCT-Ciência Animal.

2 Departamento de Zootecnia, Universidade Federal de Viçosa, Viçosa-MG, 36570-000. Pesquisador do CNPq e do INCT-Ciência Animal.

${ }^{3}$ Programa de Pós-Graduação em Zootecnia, Universidade Federal de Viçosa.
}

RESUMO - Objetivou-se neste estudo propor e discutir método de avaliação da eficiência de conversão do alimento em produto em bovinos de corte com base na utilização de técnicas de regressão. Os procedimentos matemáticos e estatísticos foram desenvolvidos a partir de banco de dados formado pela mensuração do consumo de matéria seca e do ganho médio diário em 380 bovinos zebuínos puros ou mestiços em 15 diferentes experimentos constantes na base de dados do sistema BR-CORTE Foram propostas duas aproximações baseadas no ajustamento de modelos de regressão linear e não-linear, tendo o ganho médio diário como variável independente e o consumo de matéria seca como variável dependente. A utilização do alimento pelo anima foi estratificada em demanda para mantença e eficiência real de conversão em produto. O parâmetro demanda para mantença possui interpretação similar em ambas as aproximações. Na solução linear, assume-se que a eficiência real de conversão em produto seja fixa e independente do ganho médio diário. Por outro lado, na solução não-linear, assume-se que a eficiência real de conversão em produto seja variável e dependente do ganho. O critério de informação de Akaike foi proposto como ferramenta para escolha entre os modelos linear e não-linear. A avaliação da eficiência de transformação do alimento em produto em bovinos de corte por meio de técnicas de regressão possibilita o entendimento mais amplo da eficiência de uso do alimento em sistemas de produção, pois permite estratificar as demandas em mantença e produção. Muitos inconvenientes associados ao uso de índices baseados em razões, como a conversão alimentar, são eliminados, ampliando a acurácia e a precisão das inferências nutricionais.

Palavras-chave: conversão alimentar, critério de Akaike, regressão linear, regressão não-linear

\section{Use of regression techniques in the evaluation, in beef cattle, of feed conversion into product: proposition of method and nutritional significance}

ABSTRACT - The objective of this study was to propose and discuss a method to evaluate the efficiency of feed conversion into product from beef cattle based on regression techniques. The mathematical and statistical procedures were developed using a database composed of measurements of dry matter intake and average daily gain of 380 pure or crossbred zebu animals in 15 different experiments in the database from the BR-CORTE nutritional system. Two approaches were presented, based on linear and non-linear regression, with average daily gain and dry matter intake used as independent and dependent variables, respectively. The feed utilization by the animal was stratified into maintenance demand and real efficiency of conversion into product. The maintenance demand presents similar interpretation in both approaches. When linear solution is considered, the real efficiency of product conversion is assumed to be steady and independent on average daily gain. On the other hand, when the non-linear solution is adopted, the real efficiency of product conversion is assumed to be variable and dependent on gain. Alaike information criterion was proposed to decide which model suits best. The evaluation of the efficiency of transformation of feed into product, in beef cattle, by regression techniques allows a broader understanding of system efficiency for it enables stratification of maintenance and production demands. Severa inconvenient aspects concerning ratio indexes, such as food conversion ratio, are avoided and the nutritional inferences become more accurate and precise.

Key Words: Akaike information criterion, food conversion ratio, linear regression, non-linear regression 


\section{Introdução}

As inferências sobre os fatores nutricionais e produtivos avaliados em diferentes estudos e em sistemas de produção de carne bovina demandam, normalmente, que se indique a eficiência com que o processo de construção de produto animal é realizado, ou seja, a eficiência com que o input de recursos alimentares (normalmente representado pelo consumo voluntário de matéria seca, CMS) define o output do sistema de produção (comumente representado pelo ganho médio diário, GMD).

De forma rotineira, um indicador dessa eficiência é produzido pela ponderação do CMS por unidade de GMD, índice denominado conversão alimentar (CA). Todavia, vários inconvenientes estatísticos e zootécnicos são apontados na utilização da CA, o que pode comprometer a confiabilidade das inferências obtidas a partir deste índice (Guidoni, 1994; Detmann et al., 2005). Entre outros aspectos, a variável originada da razão de duas variáveis normalmente distribuídas (CMS e GMD), neste caso a CA, demanda o atendimento de condições rigorosas para que sua avaliação estatística por análises paramétricas convencionais seja confiável (Weil Junior, 1962; Detmann et al., 2005).

Para contornar este inconveniente, tem-se proposto a avaliação simultânea do CMS e do GMD por meio de combinação linear, o que produz nova variável que preserva a característica de distribuição normal e engloba as propriedades discriminatórias das variáveis originais (Guidoni, 1994; Euclides Filho et al., 2002; Detmann et al., 2005). Neste contexto, a partir do método multivariado de variáveis canônicas, produz-se a variável denominada índice bionutricional (Detmann et al., 2005).

As vantagens estatísticas do uso do índice bionutricional em relação à CA são evidentes (Guidoni, 1994). Contudo, do ponto de vista experimental e produtivo, o índice bionutricional possui o inconveniente de seu valor ser dependente da estrutura da matriz residual dos dados do experimento avaliado (Detmann et al., 2005). Em outras palavras, para cada conjunto de dados avaliado, produzir-se-á uma função canônica diferente, o que implicará em contradomínio matemático diferente no qual se colocam as estimativas do índice bionutricional. Neste contexto, tornam-se impraticáveis a interpretação direta de seu significado numérico, a comparação entre estudos utilizando-se este valor numérico e sua utilização como informação para programas de seleção e melhoramento genético animal.

Consumo e ganho de peso são variáveis correlacionadas em um sistema de produção animal, pois o output (GMD) é dependente do input (CMS). Por outro lado, considerando-se oferta irrestrita de alimentos, o input (CMS) representa a intensidade do output (GMD). Esse comportamento pode ser utilizado diretamente para interpretação da eficiência de conversão do alimento em produto via técnicas estatísticas que permitam explorar e interpretar as propriedades desta associação, como técnicas de regressão.

Dessa forma, estabeleceram-se como objetivos neste estudo propor e discutir método de avaliação, em bovinos de corte, da eficiência de conversão do alimento em produto com base na utilização de técnicas de regressão linear e não-linear.

\section{Material e Métodos}

Os procedimentos matemáticos e estatísticos foram desenvolvidos sobre banco de dados formado pela mensuração do CMS e GMD em 380 bovinos zebuínos puros ou mestiços em 15 diferentes experimentos constantes na base de dados do sistema BR-CORTE (Valadares Filho et al., 2010). No conjunto de dados, foram avaliados animais machos não-castrados, machos castrados e fêmeas em confinamento utilizando-se fenos (Cynodon sp. ou Brachiaria sp.), forragens pré-secas (Cynodon sp. ou Brachiaria sp.), silagens (milho ou cana-de-açúcar) ou cana-de-açúcar in natura (Tabela 1; Figura 1).

A avaliação dos dados e os métodos propostos e discutidos foram desenvolvidos utilizando-se técnicas de regressão linear (Draper \& Smith, 1966; Myers, 1990) e

Tabela 1 - Caracterização das variáveis quantitativas do banco de dados (n = 380)

\begin{tabular}{|c|c|c|c|c|c|c|}
\hline \multirow[t]{2}{*}{ Item } & \multicolumn{6}{|c|}{ Estatística } \\
\hline & Média & Mediana & Moda & Máximo & Mínimo & $\mathrm{s}$ \\
\hline Nível de concentrado na dieta (\%) & 47,7 & 46,4 & 50,0 & 99,0 & 5,0 & 17,3 \\
\hline Ganho médio diário (kg/dia) & 1,08 & 1,08 & 1,21 & 1,97 & 0,27 & 0,30 \\
\hline Consumo de matéria seca (kg/dia) & 7,99 & 7,75 & 8,22 & 12,53 & 3,21 & 1,61 \\
\hline Peso corporal médio $(\mathrm{kg})^{1}$ & 367,2 & 383 & 394,3 & 498,5 & 166,5 & 60,8 \\
\hline Conversão alimentar (kg/kg) & 7,74 & 7,35 & - & 21,22 & 4,25 & 2,06 \\
\hline Dias em avaliação & 112,8 & 106 & 84 & 232 & 42 & 35 \\
\hline
\end{tabular}

${ }^{1}$ Peso corporal médio durante a avaliação. 


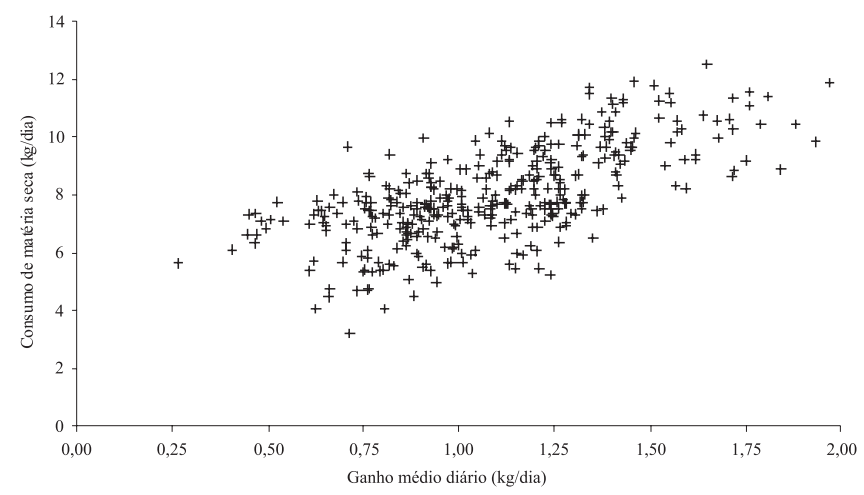

Figura 1 - Dispersão do consumo de matéria seca em função do ganho médio diário para o conjunto de dados avaliados $(\mathrm{n}=380)$.

não-linear (Bard, 1974; Souza, 1998). Os ajustamentos de modelos de regressão linear foram realizados com base no método dos mínimos quadrados ordinários e os de modelos de regressão não-linear basearam-se no método de mínimos quadrados não-lineares com o uso do algoritmo iterativo de Gauss-Newton. As análises foram conduzidas por intermédio do programa SAS (PROC REG e PROC NLIN, Statistical Analysis System, versão 9.1, licenciado para a Universidade Federal de Viçosa).

Seja Y uma variável que deve ser predita ou explicada por meio de um conjunto de fatores $\mathrm{X}_{1}, \mathrm{X}_{2}, \ldots, \mathrm{X}_{\mathrm{p}}$. Dessa forma, faz-se:

$Y=f\left(X_{1}, X_{2}, \ldots, X_{p}, \psi\right)$ em que $\psi$ representa o complemento do conjunto de fatores responsáveis pela explicação ou predição de Y não considerados ou ainda não conhecidos.

Percebe-se por intermédio da equação (1) que cada fator responde parcialmente pela explicação do comportamento de Y. A função descrita em (1) deve satisfazer ao menos quatro requisitos básicos: (I) deve ser matematicamente tratável; (II) deve ser adequada ao conjunto de dados em estudo; (III) deve ser simples ou pelo menos a mais simples entre as possíveis funções (Searle, 1971); e (IV) deve apresentar coerência e consistência com o sistema biológico descrito.

Considerando o CMS como variável-resposta a ser investigada no sistema biológico representado pelo animal em produção, pode-se pressupor que parte da explicação do comportamento desta variável possa ser atribuída ao seu nível de produção ou GMD; pois, dadas as mesmas condições de genética animal e recursos alimentares, deve ser observada relação direta e positiva entre o nível de produção (GMD) e o CMS. Assim, reescrevendo-se (1) com base nestes pressupostos, faz-se:
$\mathrm{Y}=f(X, \psi)$

em que: $\mathrm{Y}=\mathrm{CMS}$; e $\mathrm{X}=\mathrm{GMD}$.

Considerando somente a explicação proporcionada pelo GMD, tem-se:

$Y=f(X)$

A função descrita em (3) pode ser estabelecida de forma linear ou não-linear em seus parâmetros. O modelo linear geral aplicado à solução de (3) é dado por:

$Y=X \beta+\varepsilon$

em que: $\beta$ = vetor de parâmetros lineares da função; e $\underset{\sim}{\varepsilon}=$ vetor de erros não-observáveis pressupostos de distribuição normal.

Por outro lado, o modelo não-linear geral aplicado à solução de (3) é dado por:

$Y=X \underline{\theta}+\epsilon$

em que: $\underset{\sim}{\theta}$ = vetor de parâmetros não-lineares da função; e $\epsilon=$ vetor de erros não-observáveis pressupostos de distribuição normal assintótica.

Com a aplicação de métodos de mínimos quadrados lineares e não-lineares para estimação de parâmetros, as equações (4) e (5) passam a ser representadas, respectivamente, por:

$\hat{\sim}=X \hat{\beta}$

$\hat{Y}=X \hat{\theta}$

Nos métodos aqui propostos, duas situações distintas são abordadas para a eficiência biológica dos animais: fixa ou variável.

A consideração de eficiência fixa se baseia no pressuposto de que a eficiência de conversão de alimento em produto não é afetada pelo nível de produção, ou seja, atribui-se linearidade intrínseca ao processo de construção de produtos animais. De forma lógica, considerando esse pressuposto, o tratamento matemático deve ser dado utilizando-se um sistema linear (equação 6). Relevando-se as condições ditadas pelos requisitos (III) e (IV) apresentados anteriormente, apresenta-se a solução linear: $C \hat{M} S=f(X) \Rightarrow f(X)=\hat{Y} \Rightarrow \hat{Y}=\hat{\beta_{0}}+\hat{\beta_{1}} \times X$

Uma das principais limitações biológicas associadas ao uso de variáveis produzidas por razões, como a CA, reside no fato de o cômputo da eficiência considerar que todo e qualquer alimento ingerido é utilizado para construção de produto (por exemplo, GMD). Isso desconsidera, erroneamente, o fato de porção significativa dos nutrientes e atributos nutricionais do alimento ingerido ser deslocada para o suprimento dos requisitos de mantença (Detmann et al., 2005).

A partir da utilização de técnicas de regressão propõe-se a interpretação da eficiência de conversão alimento:produto a partir de dois diferentes parâmetros: demanda para 
mantença (DM) e eficiência real de conversão em produto (ERCP).

Define-se como DM a porção da matéria seca total ingerida que é necessária para manutenção das funções basais do animal sem que haja mobilização de reservas (perda de peso) ou construção de produto (ganho de peso). Desta forma, DM representa a porção do alimento ingerido que efetivamente não pode ser contabilizada como substrato para síntese de produto. Sendo um parâmetro definido em condição de variação de peso corporal nula, o estimador de DM é dado para o caso do modelo linear por:

$$
D M_{L}=\lim _{X \rightarrow 0} f(X)=\hat{\beta}_{0}
$$

em que $\mathrm{DM}_{\mathrm{L}}$ é a demanda para mantença para o caso de utilização de modelo linear.

Por outro lado, define-se como ERCP a eficiência de conversão do alimento em produto obtida após o suprimento das demandas para manutenção das atividades e metabolismo basais. Em outras palavras, ERCP somente releva a eficiência de produção a partir da porção teórica do alimento ingerido que efetivamente pode ser considerada para síntese de tecidos (crescimento). Assim, matematicamente o estimador de ERCP para o caso do modelo linear é dado por:

$E R C P_{L}=\frac{d f(X)}{d X}=\frac{d \hat{Y}}{d X}=\hat{\beta}_{1}$

em que $\mathrm{ERCP}_{\mathrm{L}}$ é a eficiência real de conversão em produto para o caso de utilização de modelo linear.

Considerando o requisito (IV), a coerência biológica e nutricional da descrição por intermédio do modelo linear somente é garantida impondo-se as restrições:

$D M>0 \Leftrightarrow \hat{\beta}_{0}>0$

$D M>0 \Leftrightarrow \hat{\beta}_{1}>0$

As restrições definidas em (11) indicam que em toda e qualquer situação produtiva sempre haverá necessidade de se suprirem demandas de mantença do animal (11a) e que qualquer substrato capaz de fornecer nutrientes e atributos nutricionais ao animal pode ser potencialmente utilizado para síntese de produto (11b) depois de supridas as demandas de mantença.

Contudo, a suposição de eficiência fixa tem sido questionada sob o ponto de vista biológico (Marcondes et al., 2010). Esses argumentos se baseiam no fato de que a eficiência de conversão de alimento em produto reduz a medida que se amplia a intensidade do processo produtivo, processo associado à lei dos rendimentos decrescentes (Lana et al., 2005). Dessa forma, a eficiência seria definida como uma função do nível de produção, caracterizando função não-linear em seus parâmetros. A partir desses argumentos, e considerando-se os requisitos (III) e (IV) apresentados anteriormente, propõe-se a solução nãolinear:

$C \hat{M} S=f(X) \Rightarrow f(X)=\hat{Y} \Rightarrow \hat{Y}=\hat{\theta}_{0} \times e^{\hat{\theta}_{1} \times X}$

Utilizando-se o conceito de partição de eficiência apresentado para a solução linear, estimam-se DM e ERCP na solução não-linear como:

$D M_{N L}=\lim _{X \rightarrow 0} f(X)=\hat{\theta}_{0}$

$E R C P_{N L}=\frac{d f(X)}{d X}=\frac{d \hat{Y}}{d X}=\hat{\theta}_{1} \times \hat{\theta}_{0} \times e^{\hat{\theta}_{1} \times X}$

em que: $\mathrm{DM}_{\mathrm{NL}}=$ demanda para mantença para o caso de utilização de modelo não-linear; e ERCP ${ }_{\mathrm{NL}}=$ eficiência real de conversão em produto para o caso de utilização de modelo não-linear.

Sob os mesmos argumentos apresentados para a equação (11), impõem-se as restrições à aplicação do modelo não-linear: $D M>0 \Leftrightarrow \hat{\theta}_{0}>0$ $E R C P>0 \Leftrightarrow \hat{\theta}_{0}>0$ e $\hat{\theta}_{1}>0$

Em função de diferenças quanto às aproximações estatísticas aplicadas a modelos lineares e não-lineares, a variância da relação entre GMD e CMS deve ser estimada pelo método da máxima verossimilhança, cujo estimador é definido por:

$s_{X Y}^{2}=\frac{S Q R}{n}=\frac{\sum_{i=1}^{n} e_{i}^{2}}{n}=\frac{\sum_{i=1}^{n}\left(Y_{i}-\hat{Y}\right)^{2}}{n}$

$s_{X Y}=\sqrt{s_{X Y}^{2}}$

A partir da estimativa de máxima verossimilhança da variância da relação entre GMD e CMS, sugere-se o cômputo do critério de informação de Akaike (AIC), o qual constitui procedimento relativo para identificação do melhor modelo de ajustamento aos dados, o qual pondera para o número de parâmetros e busca tornar a avaliação isenta de ambiguidades associadas a testes de hipóteses convencionais (Akaike, 1974).

Para o caso de modelos de regressão, o AIC pode ser estimado por (Kaps \& Lamberson, 2004):

$A I C=n \times \ln \left(s_{X Y}^{2}\right)+2 \times p$ em que p representa o número de parâmetros ajustados independentemente no modelo.

\section{Resultados e Discussão}

O banco de dados estabelecido $(n=380)$ foi utilizado para demonstração dos métodos propostos. Essa demonstração foi conduzida assumindo-se a pressuposição de os animais constituírem um grupo homogêneo e que um único processo de estimação de eficiência seria necessário para descrição da amostra. Para melhor cotejamento, a avaliação da eficiência foi realizada comparativamente 
com a CA, utilizando-se a estimativa média do banco de dados avaliado (Tabela 1).

O ajustamento dos modelos linear e não-linear e a CA média produziram, respectivamente, as estimativas ( \pm erro-padrão):

$$
\hat{C M S}=4,085( \pm 0,229)+3,604( \pm 0,205) \times G M D \quad\left(\mathrm{~s}_{\mathrm{XY}}=1,202\right)
$$

$$
C \hat{M} S=4,886( \pm 0,143) \times e^{0,4455( \pm 0,0244) \times X} \quad\left(\mathrm{~s}_{\mathrm{XY}}=1,195\right)
$$

$$
\hat{C M S}=7,74( \pm 0,091) \times G M D \quad\left(\mathrm{~s}_{\mathrm{XY}}=1,771\right)
$$

A aplicação do conceito de DM aos modelos ajustados (equações 19 e 20) fornece as estimativas:

$D M_{L}=\lim _{X \rightarrow 0} f(X)=4,085$

$D M_{N L}=\lim _{X \rightarrow 0} f(X)=4,886$

O parâmetro DM apresenta a mesma interpretação, tanto na aproximação linear como não-linear e corresponde à porção da matéria seca total ingerida (kg/dia) necessária para a manutenção do metabolismo e atividades basais dos animais. Essa estratificação permite a identificação de grupos genéticos ou situações alimentares que possam apresentar discrepâncias quanto a este aspecto nutricional. A divergência quanto às estimativas produzidas pelos modelos linear e não-linear (equações 22 e 23) são verossímeis em função da estrutura diferenciada destes modelos (equações 8 e 12; Figura 2). A decisão sobre qual das estimativas deverá ser adotada será discutida posteriormente em conjunto com o parâmetro ERCP.

Em termos comparativos, os modelos linear e nãolinear (Figura 2) expressaram coerentemente a tendência demonstrada pelo conjunto de dados avaliado (Figura 1). No entanto, a relação entre CMS e GMD descrita pela CA (equação 21) somente foi coerentemente descrita para os animais com desempenho correspondente à média do grupo

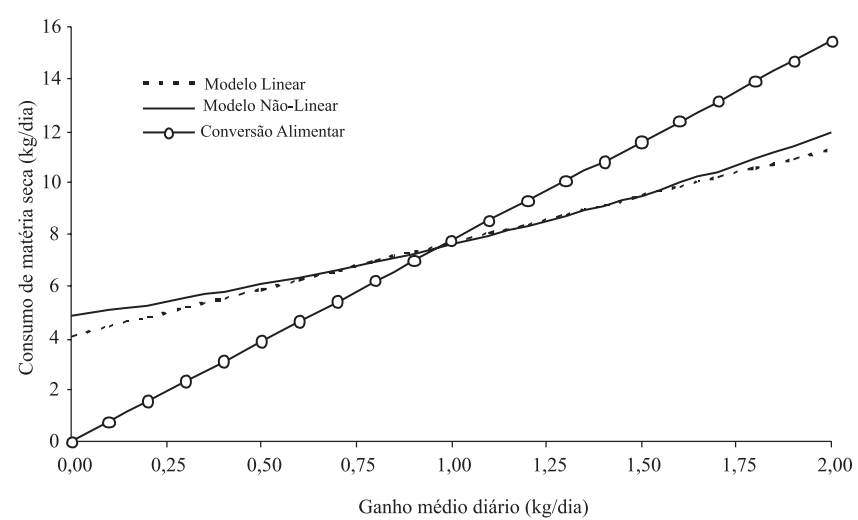

Figura 2 - Relação entre consumo de matéria seca e ganho médio diário predita pelos modelos linear (equação 19) e nãolinear (equação 20) e pelo valor médio de conversão alimentar (equação 21).
(Tabela 1; Figura 2). A relação ditada pela estrutura matemática da CA (razão CMS/GMD) assume forma retilínea, passando pela origem (equação 21; Figura 2), o que provoca o deslocamento da curva ajustada em relação aos valores observados, subestimando e superestimando o CMS para animais com GMD abaixo e acima da média do grupo, respectivamente. De forma mais específica, animais com CMS e GMD abaixo da média de seus pares apresentariam GMD positivos mesmo com consumo teoricamente abaixo das necessidades de mantença (Figura 2). Essa incoerência nutricional ditada pela utilização da CA pode ser percebida aplicando-se o conceito de DM à equação (21):

$D M_{C A}=\lim _{X \rightarrow 0} f(X)=0$

em que $\mathrm{DM}_{\mathrm{CA}}$ é a demanda para mantença para o caso de utilização da CA.

Assim, a utilização da CA para avaliação da eficiência de transformação de alimento em produto pressupõe implicitamente que não há demanda para mantença animal (Detmann et al., 2005), o que é incoerente dos pontos de vista nutricional e produtivo (equação 24).

Adicionalmente, a estrutura da relação entre GMD e CMS dada pela CA, em função do viés anteriormente ressaltado, apresenta desvios elevados nas observações afastadas da média do grupo, o que reduz a precisão da avaliação em comparação ao uso de equações de regressão. Isso pode ser diretamente percebido pelas estimativas dos desvios-padrão residuais mostrados nas equações 19, 20 e 21.

De forma geral, outras incoerências nutricionais podem ser verificadas sobre a CA, as quais são demonstradas por:

$C A=f(X, Y)=\frac{Y}{X}$

$\lim f(X, Y)_{X \rightarrow 0}=\infty$

$f(X, Y)<0 \forall X<0$

em que: $\mathrm{X}=\mathrm{GMD}$; e $\mathrm{Y}=\mathrm{CMS}$.

A incoerência evidenciada pela equação (26) indica que a CA converge à indeterminação matemática no caso de animais mantidos em situação de mantença (GMD $=0$ ), o que é reflexo da ausência de estratificação entre demandas de mantença e produção. Adicionalmente, sob situações de ganho negativo (perda de peso), entende-se que o consumo de alimento pelo animal encontra-se aquém do necessário para sua manutenção. Contudo, a interpretação dessa situação por intermédio da CA evidencia que a eficiência animal é negativa, demandando consumo negativo para determinada situação, uma vez que sua interpretação direta indica qual a quantidade de matéria seca a ser ingerida por unidade de GMD (equação 27).

A aplicação dos conceitos de ERCP aos modelos ajustados (equações 19 e 20) fornece as estimativas: 
$E R C P_{L}=\frac{d f(X)}{d X}=\frac{d \hat{Y}}{d X}=3,604$

$E R C P_{N L}=\frac{d f(X)}{d X}=\frac{d \hat{Y}}{d X}=2,177 \times e^{0,4455 \times X}$

A conceituação teórica de ERCP baseia-se na demanda real de alimento por unidade de GMD (kg MS/kg GMD) considerando que as exigências de mantença tenham sido atendidas. Logo, o parâmetro ERCP representa as demandas variáveis de alimento para produção, pois estas estão relacionadas à intensidade de ganho. Assim, o parâmetro DM representa a demanda fixa de alimentos para o sistema de produção, pois indica as necessidades para manutenção de atividades e metabolismo basais do animal.

Embora esteja direcionada à descrição da demanda real para construção de produto (GMD), a conceituação matemática de ERCP difere nas aproximações linear e nãolinear. O parâmetro ERCP estimado por modelo linear assume que, depois de supridas as exigências de mantença, a eficiência de conversão do alimento em produto é fixa e independente do nível de produção (equações 10 e 28). Por outro lado, a aproximação não-linear assume que a ERCP varia de acordo com o nível de produção (equações 14 e 29), havendo redução gradativa da eficiência (maior demanda de CMS) com a elevação de GMD (Figura 3).

A consideração do parâmetro ERCP na avaliação de grupos genéticos ou situações alimentares permite agregar discriminação quanto à capacidade real de transformação de alimento em produto animal, pois estratifica apenas a porção variável da produção (ganho). Este aspecto é comprometido com o uso da CA, uma vez que demandas fixas (mantença) e variáveis (ganho) para produção não são estratificadas, o que reduz o entendimento sobre o processo de construção de produto animal e subestima a eficiência produtiva animal. Neste caso, a eficiência de transformação do alimento em produto dada pela CA deve ser considerada aparente, pois, ao assumir-se que não há DM (equação 24), todo o custo alimentar para construção de produto é assumido como sendo variável. Assim, a eficiência é subestimada (Figura 3), como demonstrado a seguir:

$$
E A C P_{C A}=\frac{d f(X)}{d X}=\frac{d \hat{Y}}{d X}=7,74
$$

em que $\mathrm{EACP}_{\mathrm{CA}}$ é a eficiência aparente de conversão em produto dada pela CA (kg CMS/kg GMD).

Em termos estritamente heurísticos, para melhor compreensão do conceito de conversão do alimento em produto, pode-se proceder à predição do produto animal (GMD) a partir da recíproca dos modelos anteriormente apresentados, o que teoricamente é dado por:

$$
\begin{aligned}
& C M S=D M+E R C P \times G M D \Rightarrow G M D=\frac{C M S-D M}{E R C P} \\
& C M S=D M \times e^{\hat{\theta}_{1} \times G M D} \Rightarrow G M D=\frac{\ln C M S-\ln D M}{\hat{\theta}_{1}} \\
& C A=\frac{C M S}{G M D} \Rightarrow G M D=\frac{C M S}{C A} \Rightarrow G M D=\frac{1}{C A} \times C M S
\end{aligned}
$$

A utilização do banco de dados (equações 19, 20 e 21) confere as estimativas:

$G M D=\frac{C M S-4,085}{3,604}$

$G M D=\frac{\ln C M S-1,5864}{0,4455}$

$G M D=\frac{1}{7,74} \times C M S$

Verifica-se que tanto a aproximação linear (equações 31 e 34) como a não-linear (equações 32 e 35) consideram que a construção de produto somente pode ser relevada após o suprimento das demandas de mantença. Logo, caso o CMS encontre-se abaixo de DM, haverá perda de peso (Figura 4). De outra forma, a CA, por não relevar demandas para mantença (equações 33 e 36), associa GMD nulo a CMS nulo (Figura 4). Em outras palavras, perdas de peso são matematicamente obtidas somente com CMS negativo, o que representa claramente incoerência biológica e nutricional.

Embora a opção por ERCP fixa ou variável (o que equivale ao uso de modelo linear, equação 8; ou não-linear, equação 12; respectivamente) possa ser suportada pela interpretação biológica utilizada pelo pesquisador em determinada situação, sugere-se que a escolha seja realizada com base na qualidade relativa do ajustamento dos modelos aos dados.

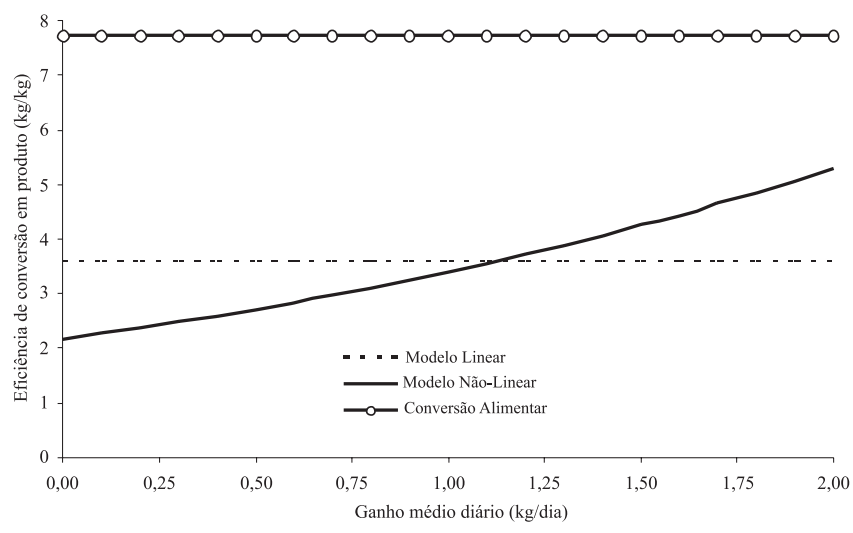

Figura 3 - Eficiências reais de conversão em produto (kg de matéria seca consumida/kg de ganho médio diário) preditas pelos modelos linear (equação 28) e não-linear (equação 29) e eficiência aparente de conversão em produto (kg matéria seca consumida/kg de ganho médio diário) dada pelo valor médio de conversão alimentar (equação 30). 
Entre os dois modelos aqui propostos (equações 8 e 12), aquele que apresentar a menor estimativa de AIC deve ser considerado mais adequado para descrição da relação entre CMS e GMD. Para o caso específico do conjunto de dados aqui avaliado, o modelo não-linear foi considerado mais adequado (Tabela 2). Logo, o conjunto de dados (Figura 1) deve ser interpretado considerando ERCP variável em função do GMD (Figura 3).

Em primeira instância, o cômputo do AIC pode parecer redundante, uma vez que ambos os modelos apresentam dois parâmetros (equações 8 e 12). Assim, a avaliação do ajustamento poderia ser realizada diretamente por intermédio

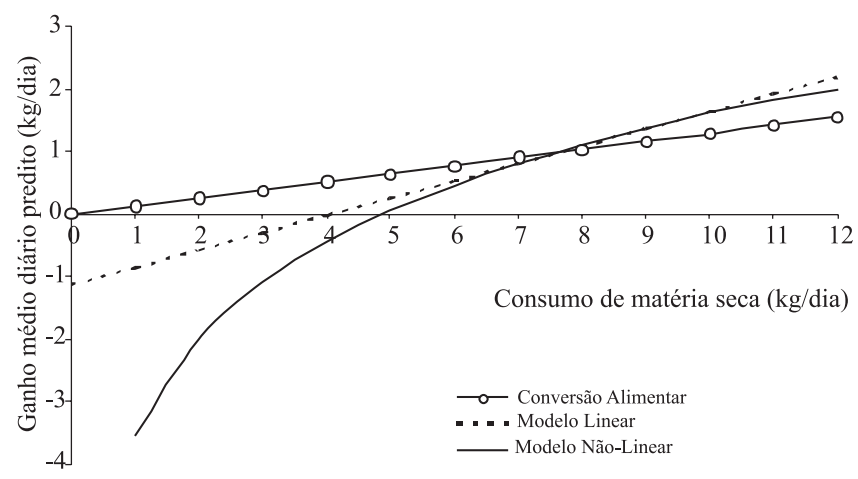

Figura 4 - Predição do ganho médio diário por intermédio das recíprocas dos modelos linear (equação 34) e nãolinear (equação 35) e da conversão alimentar média (equação 36). da variância ou desvio-padrão residual (equações 16 e 17; Tabela 2). Contudo, essa situação avaliada deve ser vista apenas como demonstrativa. Na maioria dos casos, diferentes dietas e/ou grupos genéticos podem ser comparados, e os modelos linear e não-linear ajustados considerando-se as comparações poderão apresentar número diferente de parâmetros, uma vez que diferenças quanto à DM e/ou à ERCP podem ser detectadas por somente um dos modelos (Detmann et al., 2011). Isso torna o AIC o critério mais acurado para escolha da melhor solução em todas as situações.

Apesar do embasamento matemático e nutricional demonstrados, duas limitações são observadas na avaliação da eficiência de transformação do alimento em produto quando utilizadas técnicas de regressão.

Em primeiro lugar, o ajustamento dos modelos depende do conjunto de dados. Quanto maior a variabilidade em $\mathrm{X}$ (neste caso GMD), maior a confiabilidade do modelo ajustado (Draper \& Smith, 1966). Logo, limitações quanto às estimativas de DM e ERCP podem ser observadas em amostras de tamanho reduzido, podendo ocorrer situações em que as restrições necessárias à aplicação da técnica não sejam estabelecidas (equações 11 e 15).

Em segundo lugar, a variável proposta como variável independente (GMD; equações 8 e 12) constitui variável aleatória e, portanto, está sujeita a erro experimental. Logo, incrementos estatísticos nas estimativas de DM e ERCP poderão ser obtidos considerando o erro inerente ao GMD no processo de estimação (Bartlett, 1949).

Tabela 2 - Avaliação da eficiência de descrição da relação entre consumo de matéria seca e ganho médio diário por diferentes métodos (equações 19, 20 e 21)

\begin{tabular}{cccc}
\hline & & Método & \\
\cline { 2 - 4 } Item $^{1}$ & Modelo linear & Modelo não-linear & Conversão alimentar \\
\hline $\mathrm{s}_{\mathrm{XY}}$ & 1,202 & 1,195 & 1,771 \\
$\mathrm{p}$ & 2 & 2 & 1 \\
AIC & 143,98 & 139,16 & 436,44 \\
\hline
\end{tabular}

${ }^{1} \mathrm{~s}_{\mathrm{XY}}$ = desvio-padrão residual; $\mathrm{p}$ = número de parâmetros utilizados para descrição da relação; AIC = critério de informação de Akaike.

\section{Conclusões}

A avaliação da eficiência de transformação do alimento em produto em bovinos de corte por intermédio de técnicas de regressão confere entendimento amplo da eficiência de uso do alimento em sistemas de produção, pois permite a estratificação das demandas para mantença e produção. Muitos inconvenientes associados ao uso de índices baseados em razões, como a CA, são eliminados, ampliando a acurácia e a precisão das inferências nutricionais.

\section{Referências}

AKAIKE, H. A new look at the statistical model identification. IEEE Transactions on Automatic Control, v.19, p.716-723, 1974.

BARD, Y. Nonlinear parameter estimation. New York: Academic Press, 1974. 341p.

BARTLETT, M.S. Fitting a straight line when both variables are subject to error. Biometrics, v.5, p.207-212, 1949.

DETMANN, E.; CECON, P.R.; ANDREOTTI, M.O. et al. Application of the first canonical variable in the evaluation of animal production trials. Revista Brasileira de Zootecnia, v.34, p.2417-2426, 2005. 
DETMANN, E.; VALADARES FILHO, S.C.; GIONBELLI, M.P. et al. Uso de técnicas de regressão na avaliação da eficiência de conversão alimento:produto em bovinos de corte: comparação entre grupos experimentais. Revista Brasileira de Zootecnia, 2011 (no prelo).

DRAPER, N.; SMITH, H. Applied regression analysis. New York: John Willey \& Sons, 1966. 407p.

EUCLIDES FILHO, K.; FIGUEIREDO, G.R.; EUCLIDES, V.P.B. et al. Eficiência bionutricional de animais da raça nelore e seus mestiços com caracu, angus e simental. Revista Brasileira de Zootecnia, v.31, p.331-334, 2002.

GUIDONI, A.L. Alternativas para comparar tratamentos envolvendo o desempenho nutricional animal. 1994. $105 \mathrm{f}$. Tese (Doutorado em Agronomia) - Escola Superior de Agricultura "Luiz de Queiroz"/Universidade de São Paulo, Piracicaba.

KAPS, M.; LAMBERSON, W. Biostatistics for animal science. Cambridge: CAB International, 2004. 445p.

LANA, R.P.; GOES, R.H.T.B.; MOREIRA, L.M. et al. Application of Lineweaver-Burk data transformation to explain animal and plant performance as a function of nutrient supply. Livestock Production Science, v.98, p.219-224, 2005.

MARCONDES, M.I.; CHIZZOTTI, M.L.; VALADARES FILHO, S.C. et al. Energy requirements of zebu beef cattle. In: VALADARES FILHO, S.C.; MARCONDES, M.I.; CHIZZOTTI, M.L. et al. (Eds.) Nutrient requirements of zebu cattle BR-CORTE. 2.ed. Viçosa, MG: DZO-UFV, 2010. p.81-106.

MYERS, R.H. Classical and modern regression with applications. Boston: PWS-Kent Publishing Co, 1990. 488p.

SEARLE, S.R. Linear models. New York: John Willey \& Sons, 1971. 560p.

SOUZA, G.S. Introdução aos modelos de regressão linear e não-linear. Brasília: EMBRAPA-SPI, 1998. 505p.

VALADARES FILHO, S.C.; MARCONDES, M.I.; CHIZZOTTI, M.L. et al. (Eds.) Nutrient requirements of zebu cattle BR-CORTE. 2.ed. Viçosa, MG: DZO-UFV, 2010. 185p.

WEILL JUNIOR, W.B. Adjustment for size - a possible misuse of ratios. American Journal of Clinical Nutrition, v.11, p.249-252, 1962. 